\title{
Influence of International Crude Oil Price Fluctuations on the Shanghai Cooperation Organization Member Countries and Observer Countries

\author{
Yu Wang
}

School of Economics, Shanghai University, Shanghai, China

wymjhy@126.com

Keywords: Crude oil prices; PVAR; The Shanghai cooperation organization; Regional cooperation

\begin{abstract}
Based on the panel data of the Shanghai Cooperation Organization member countries and observer countries' economic growth and the exchange rate from 1994 to 2015, this paper established the panel vector auto regression model (PVAR), and analyzed how the international oil prices fluctuation affects he Shanghai Cooperation Organization member states and observer countries using impulse response and variance decomposition analysis. We found, in short-term, international oil prices promoted oil-exporting countries economic growth, and inhibited oil-importing countries. In the long term, the increasing oil prices will be beneficial to the SCO member countries on economic development. The Shanghai Cooperation Organization member countries and observer countries should expand cooperation and promote economic restructuring, in order to withstand the international crude oil market risks resulting from fluctuations.
\end{abstract}

\section{国际原油价格波动对上海合作组织相关国家经济 发展的影响}

\author{
王宇 \\ 上海大学 经济学院，中国上海 200444 \\ wymjhy@126.com
}

摘 要: 本文选取 1994-2015 年上海合作组织相关国家 GDP 和汇率的面板数据, 建立面板向量 自回归模型(PVAR), 分析国际油价波动对上海合作组织相关国家经济发展的影响。分析发现, 短期内国际油价上涨对上合组织中原油出口国经济增长具有明显的促进作用, 对原油进口国 经济增长具有抑制作用, 长期来看, 油价上涨有利于上合组织相关国家经济发展。上海合作 组织相关国家应积极推动区域内各领域的合作, 促进各国经济结构调整, 增强抵御国际原油 市场波动所造成的风险的能力。

关键词：原油价格; PVAR; 上海合作组织; 区域合作

\section{1. 引言}

随着 “一带一路” 战略的快速推进, 无论从地缘角度还是经贸角度出发, 上海合作组织处于 “丝绸之路经济带”的黄金地段，其重要性日渐突出。上海合作组织成员以及观察员国经贸 关系的紧密程度以及各国经济的稳定性对 “一带一路”战略的顺利实施具有直接的影响。从 上合组织各相关国家的经济结构和贸易结构来看, 能源尤其是石油和天然气扮演着重要角色。 2014 年面对国际原油市场价格大幅下滑，上海合作组织成员国与观察员国经济都面临着考验 和挑战, 从中长期来看, 如何应对国际原油价格危机, 利用上海合作组织内部的合作机制, 建立及强化区域内经贸合作关系，抓住 “一带一路” 战略的合作机遇推动上海合作组织成员 国与观察员国的经贸合作，有利于发挥地区经济的互补效应，促进上海合作组织范围内各成 员国及观察员国的稳定增长和贸易一体化进程。 


\section{2. 计量模型与数据来源}

\section{1. 变量选择及数据来源}

本文重点分析研究国际原油价格波动与上海合作组织相关国家经济发展的关系, 在选取变量 时考虑到已有文献以及上海合作组织相关国家的实际情况, 选取三个主要变量, 一是国际原 油价格, 单位: 美元/桶, 采用美国西德克萨斯轻质原油价格（West Texas Intermediate，简称 WTI）代表国际原油价格, 数据来自美国能源部信息署网站。二是各国国内生产总值, 单位: 美元, 以上一年为基期, 用以测度每个国家的经济增长情况, 数据来源于世界银行在线数据 库。三是本国货币-美元汇率, 数据来源于世界银行在线数据库。通过汇率这一变量将外生的 国际石油价格内生化, 使之成为国内经济增长模型的内生变量。为减小数据存在的异方差性, 本文在进行实证研究时对上述变量的原始数据均取了自然对数, 处理后的各变量分别表示为: $l o p 、 l g d p$ 、 lex. 本文选取上海合作组织各成员国（中国、俄罗斯、哈萨克斯坦、吉尔吉斯斯 坦、塔吉克斯坦和乌兹别克斯坦）和观察员国（伊朗、阿富汗、蒙古、巴基斯坦、印度）共 11 个国家 1994-2015 年的面板数据。

\section{2. 计量模型}

通常向量自回归模型（Vector Autoregression，简称 VAR 模型）要求数据时间跨度较长，方可 取得较好的效果，而各国 GDP 和对美元汇率统计数据公布的年份不满足 VAR 模型的时间跨度 要求, 特别是本国货币-美元汇率数据时间不足 25 年。Holtz-Eakin, Newey 和 Rosen (1998) 认为运用面板数据进行计量分析能有效克服数据量不足的问题, 但一般的 VAR 模型不适用于 面板数据, 因为面板数据中通常包含很多变量, 并且变量之间的异质性较为明显, 一般的 VAR 模型并未将这些问题考虑在内。因此, 本文采用面板向量自回归模型（Pane1 Vector Autoregression, 简称 PVAR 模型) 进行实证分析。PVAR 模型不仅拥有 VAR 模型和面板数据估 计方法的优点, 也降低了一般 VAR 模型对时间序列跨度的要求, 还可以较全面地将样本个体 差异对模型的影响考虑在内。

由于无法事先确定模型的滞后阶数, 本文估计了一到三阶滞后的 PVAR 模型, 根据 AIC 信息准 则, 确定最优滞后阶数为三阶。考虑下列 PVAR 模型:

$$
y_{i t}=A y_{i t-1}+B y_{i t-2}+C y_{i t-3}+f_{i}+\varphi_{t}+\mu_{i t}
$$

其中, $y_{i t}=\left(\operatorname{lgdp} z_{i t} ， \operatorname{lex} z_{i t} ， \operatorname{lop} z_{i t}\right),(z=1,2 ; z=1$ 代表上海合作组织中的原油进口国， $\mathrm{z}=2$ 代 表上海合作组织中的原油出口国); $\lg d p z$ 代表上海合作组织成员国与观察员国的国内生产总 值的对数, lexz 代表各国本国货币-美元的汇率的对数, lopz 代表国际原油期货价格的对数; $y_{i t-p}$ 代表 $y_{i t}$ 的 $\mathrm{p}$ 阶滞后项; $y_{i t}$ 中的 $\mathrm{i}$ 代表国家， $t$ 代表时间; $\mathrm{A} 、 \mathrm{~B} 、 \mathrm{C}$ 均是四阶系数方阵, $f_{i}$ 是 $4 \times 1$ 的个体效应向量, $\varphi_{t}$ 是 $4 \times 1$ 的时间效应向量, $\mu_{i t}$ 是随机干扰项。

由于本文建立的模型中包含个体效应和时间效应, 与实际情况不符, 因此在进行模型估计前 要先消除二者的影响。首先采用组内去心法消除时间效应, 接着采用 Arellano, Bover（1995） 的前向均值差分法消除个体效应, 以避免个体效应与回归变量间的相关性造成的估计偏差, 然后基于系统 GMM 方法进行模型估计，最后进行脉冲响应函数估计和方差分解。

\section{3. 数据来源与计量模型}

\section{1. 数据分析}

通过 Eviews 对国际原油价格与每个国家 GDP 两个变量进行了回归, 回归结果如表 1 所示。上 海合作中的大部分原油进口国 GDP 与国际原油价格呈正向关系，其中中国 GDP 与国际原油价 格的相关系数为 0.58 , 表明国际原油价格上涨对中国经济的影响呈现较为显著地正向作用。 中国作为全球最大的原油进口国, 国家经济实力的逐步提升有效抵御了原油价格上涨带来的 负面冲击。相较于中国, 蒙古作为原油出口国, 本国 GDP 与国际原油价格的相关系数为 -1.44 , 说明原油价格上涨会导致本国经济下滑。与此同时，上海合作组织中的七个原油出口国 GDP 与国际原油价格呈正向关系，其中，俄罗斯、哈萨克斯坦和伊朗国家 GDP 与国际原油价格的 
相关系数为 $0.79 、 0.67$ 和 0.61 , 上述三个国家作为具有代表性的原油出口国, 原油出口收 入在本国 GDP 中占据核心地位, 国家经济发展与国际原油价格波动密切相关。

表 1 数据回归结果

\begin{tabular}{ccccc}
\hline & 国家 & 系数 & t 值 & 结果 \\
\hline & 中国 & 0.58 & 1.13 & 显著 \\
原油进口国 & 印度 & 0.53 & 1.04 & 显著 \\
& 巴基斯坦 & 0.62 & 1.21 & 显著 \\
& 蒙古 & -1.44 & -2.83 & 显著 \\
\hline & 俄罗斯 & 0.79 & 1.54 & 显著 \\
& 哈萨克斯坦 & 0.67 & 1.31 & 显著 \\
& 吉尔吉斯斯坦 & 0.45 & 0.88 & 不显著 \\
原油出口国 & 塔吉克斯坦 & 0.37 & 0.73 & 不显著 \\
& 乌兹别克斯坦 & 0.37 & 0.73 & 不显著 \\
& 伊朗 & 0.61 & 1.20 & 显著 \\
& 阿富汗 & 0.31 & 1.35 & 显著 \\
\hline
\end{tabular}

\section{2. 模型估计结果}

根据 PVAR 模型的滞后阶数, 采用系统 GMM 方法估计得到了经济增长、汇率与原油价格之间的 PVAR 模型的参数估计结果, 如表 2 所示。分析估计表 2 的结果可知:

表 2 PVAR 估计结果

\begin{tabular}{|c|c|c|c|c|c|c|c|}
\hline 变量（原油进口国） & h_lop1 & $h \_1 g d p 1$ & h_ler1 & 变量（原油出口国） & h_lop2 & $h \_1 g d p 2$ & h_ler2 \\
\hline L. h_lopl & $\begin{array}{c}0.20 \\
(1.28)\end{array}$ & $\begin{array}{c}-0.08 \\
(-1.01)\end{array}$ & $\begin{array}{c}0.07 \\
(0.23)\end{array}$ & L. h_lop2 & $\begin{array}{c}0.32 \\
(3.62)\end{array}$ & $\begin{array}{c}0.02 \\
(1.33)\end{array}$ & $\begin{array}{c}0.37 \\
(0.12)\end{array}$ \\
\hline L. h_lgdpl & $\begin{array}{c}-0.13 \\
(-1.09)\end{array}$ & $\begin{array}{c}1.19 \\
(3.01)\end{array}$ & $\begin{array}{c}-0.03 \\
(-0.72)\end{array}$ & L. h_lgdp2 & $\begin{array}{c}-0.22 \\
(-1.33)\end{array}$ & $\begin{array}{c}0.88 \\
(3.17)\end{array}$ & $\begin{array}{c}-0.57 \\
(-1.05)\end{array}$ \\
\hline L. h_lerl & $\begin{array}{c}0.06 \\
(0.75)\end{array}$ & $\begin{array}{c}-0.81 \\
(-1.29)\end{array}$ & $\begin{array}{c}0.90 \\
(3.31)\end{array}$ & L.h_ler2 & $\begin{array}{c}0.01 \\
(1.52)\end{array}$ & $\begin{array}{c}-0.02 \\
(-1.71)\end{array}$ & $\begin{array}{c}1.22 \\
(6.06)\end{array}$ \\
\hline L2. $h \_1 o p 1$ & $\begin{array}{c}0.28 \\
(1.82)\end{array}$ & $\begin{array}{c}-0.01 \\
(-0.56)\end{array}$ & $\begin{array}{c}0.22 \\
(0.81)\end{array}$ & L2. $h \_1 o p 2$ & $\begin{array}{c}0.20 \\
(2.39)\end{array}$ & $\begin{array}{c}0.43 \\
(0.95)\end{array}$ & $\begin{array}{c}0.84 \\
(1.26)\end{array}$ \\
\hline L2. $h \_1 g d p 1$ & $\begin{array}{c}-1.04 \\
(-1.57)\end{array}$ & $\begin{array}{c}-0.07 \\
(-0.20)\end{array}$ & $\begin{array}{c}0.03 \\
(0.93)\end{array}$ & L2. h_lgdp2 & $\begin{array}{c}1.01 \\
(1.31)\end{array}$ & $\begin{array}{c}2.19 \\
(1.80)\end{array}$ & $\begin{array}{c}0.36 \\
(0.55)\end{array}$ \\
\hline L2. h_ler1 & $\begin{array}{c}0.42 \\
(0.88)\end{array}$ & $\begin{array}{c}0.01 \\
(0.01)\end{array}$ & $\begin{array}{c}-0.23 \\
(-0.83)\end{array}$ & L2. h_ler2 & $\begin{array}{l}1.01 \\
(3.01)\end{array}$ & $\begin{array}{c}0.01 \\
(1.81)\end{array}$ & $\begin{array}{c}0.42 \\
(1.75)\end{array}$ \\
\hline L3. $h \_l o p 1$ & $\begin{array}{c}0.22 \\
(1.82)\end{array}$ & $\begin{array}{c}0.08 \\
(0.34)\end{array}$ & $\begin{array}{c}0.39 \\
(0.78)\end{array}$ & L3. h_lop2 & $\begin{array}{c}0.26 \\
(4.01)\end{array}$ & $\begin{array}{c}0.49 \\
(2.12)\end{array}$ & $\begin{array}{c}4.49 \\
(1.33)\end{array}$ \\
\hline L3. $h \_1 g d p 1$ & $\begin{array}{c}0.80 \\
(0.26)\end{array}$ & $\begin{array}{c}-0.24 \\
(-1.59)\end{array}$ & $\begin{array}{l}-0.01 \\
(-0.01)\end{array}$ & L3. h_lgdp2 & $\begin{array}{c}0.68 \\
(0.14)\end{array}$ & $\begin{array}{c}0.15 \\
(0.63)\end{array}$ & $\begin{array}{c}0.06 \\
(0.15)\end{array}$ \\
\hline L3. h_ler1 & $\begin{array}{c}0.04 \\
(1.23)\end{array}$ & $\begin{array}{c}0.10 \\
(0.61)\end{array}$ & $\begin{array}{c}0.14 \\
(0.58)\end{array}$ & L3. h_ler2 & $\begin{array}{c}0.01 \\
(0.55)\end{array}$ & $\begin{array}{c}0.01 \\
(1.31)\end{array}$ & $\begin{array}{c}0.07 \\
(1.50)\end{array}$ \\
\hline
\end{tabular}

注： L、L2、L3 分别表示滞后 1、2、3 期, 括号内为 $\mathrm{t}$ 检验值。

第一，国际原油价格滞后 1、2 期对原油进口国经济增长具有显著的负面影响，滞后 3 期国际 油价对经济增长的促进作用较为明显, 国际原油价格滞后 1 期对原油出口国具有负面影响, 滞后 2、3 期国际油价对经济增长具有正面影响。而经济增长滞后 1、2、3 期对国际油价的影 响均不显著, 说明短期内国际油价上涨对原油进口国经济增长具有显著地抑制作用, 对原油 出口国经济增长具有促进作用, 但是长期内国际油价波动对各国经济增长则显现出促进作用。 国际油价上涨初期存在一系列社会调整成本，对原油进口国宏观经济是不利的，随着时间推 移，社会生产逐步调整至最佳状态，中国、印度等能源依赖型国家能够很好应对高油价带来 的风险, 并且高涨的油价增加了俄罗斯、伊朗等资源型国家的出口资源价格, 有利于各国国 内经济快速发展。

第二, 随着国际油价波动的持续, 汇率呈现由正向变动逐渐减弱的过程, 滞后 1 期呈现出不 显著的正向变动, 滞后 2 期呈现显著的正向变动, 滞后 3 期则呈现出不显著的正向变动, 反 
过来汇率滞后 1、2 期对国际油价呈现显著的正面影响, 滞后 3 期汇率对国际油价的影响趋于 平静, 呈现不显著的正面效应, 说明国际油价和汇率的之间存在正向的相互影响关系, 存在 1-2 年的滞后期, 原油出口国比原油进口国能够更加明显地体现这种关系。国际原油价格上 升造成本国货币汇率提高, 即美元贬值, 本币升值; 本国汇率下降, 即美元升值, 本币贬值, 导致国际原油价格下跌。2014 年美国退出货币量化宽松政策, 导致美元升值, 国际油价也存 在进一步下跌的趋势, 作为世界主要的石油生产和出口国, 俄罗斯卢布极容易受到国际原油 价格不断下跌与美元升值的影响, 在此情况下, 俄罗斯央行外汇储备大幅缩水, 导致外汇市 场上卢布大幅贬值, 从 2015 年初以来卢布汇率已经累计贬值 $50 \%$ 以上。

\section{3. $3 . \quad$ 脉冲响应分析}

脉冲响应函数描述的是模型中某一变量的正交化新息对模型中每一个变量当期和未来值的影 响, 能比较直观地反映出变量间的交互动态关系。图 2 和图 3 即为各变量间的 3 阶滞后脉冲 响应图, 图中的中间实线为脉冲响应点估计值, 上下两条分别为 $95 \%$ 置信区间的上下边界, 横轴表示滞后期数 (年份), 纵坐标表示对变量冲击的反应程度 (百分数)。

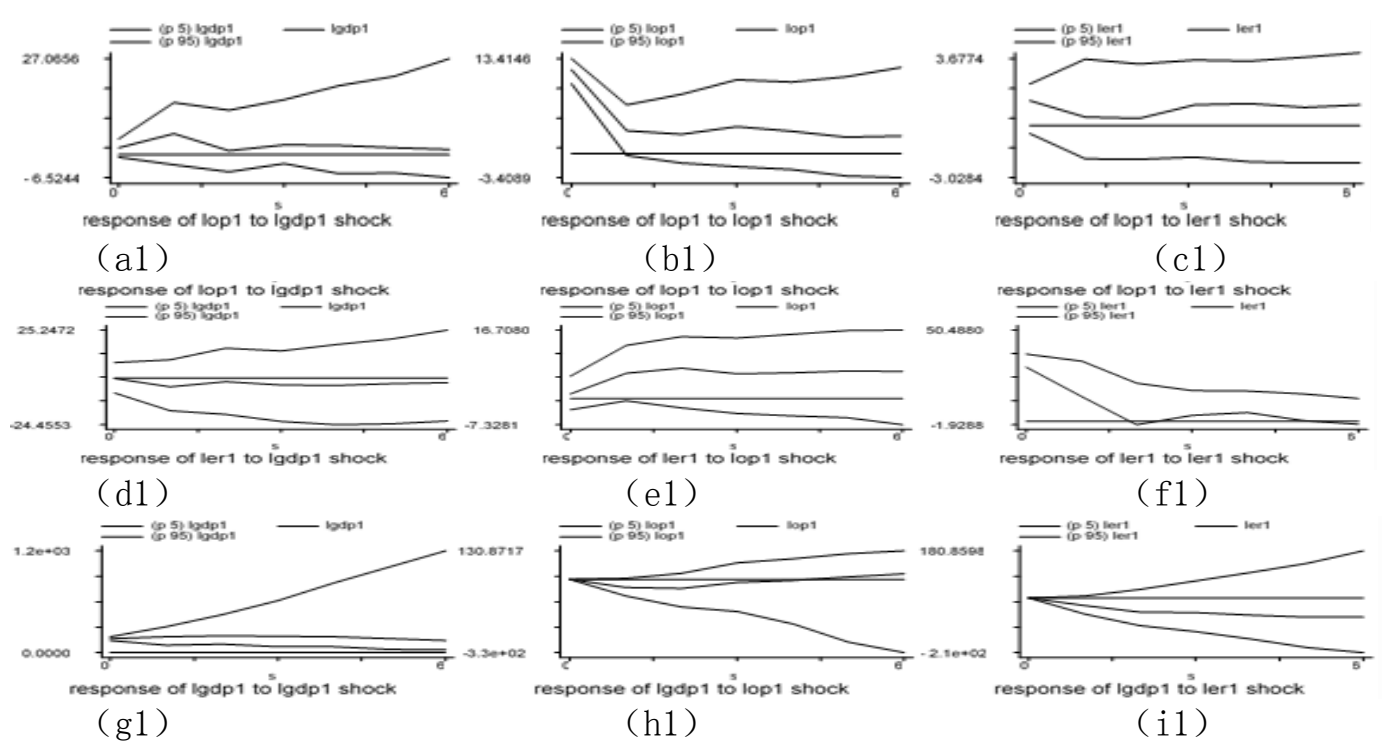

图 2-1 脉冲响应图（原油进口国）

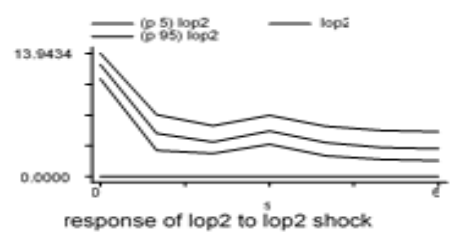

( a2)

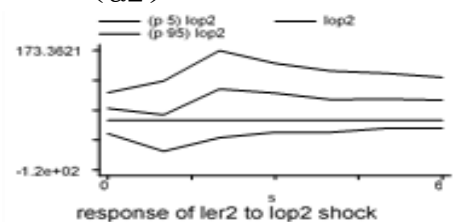

(d2)

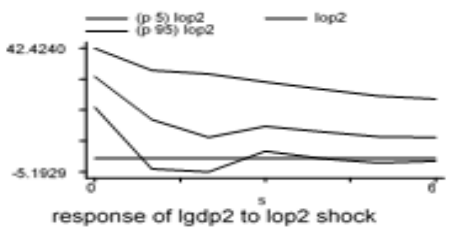

(g2)

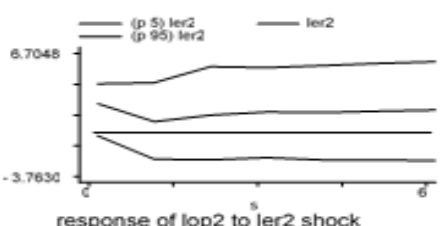

(b2)

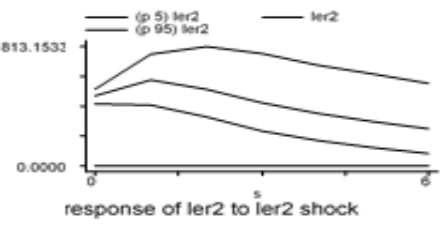

(e2)

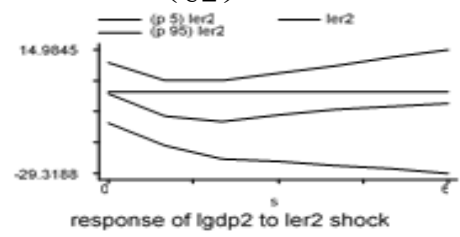

(h2)

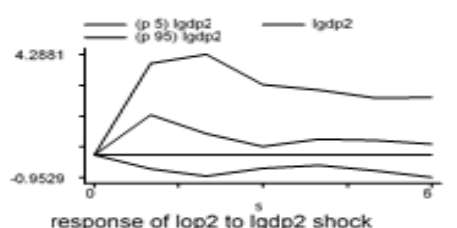

(c2)

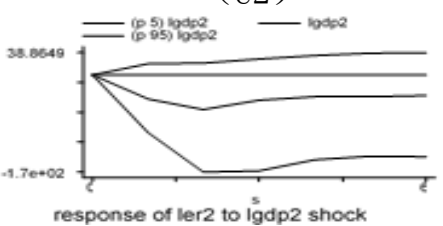

(f2)

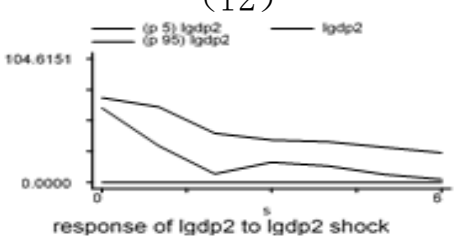

(i2)

图 2-2 脉冲响应图（原油出口国） 
第一，经济增长对国际原油价格冲击的反应。面对国际原油价格的一个正交化新息，图 2-1 中的 (h1) 显示经济增长在同期呈现负向响应, 滞后响应程度逐渐减小, 到滞后第 3 期时响 应为零, 滞后正向响应逐渐增强并趋于平稳, 一直持续到第 6 期; 图 2-2 中的（g2）显示面 对原油价格的冲击, 经济增长长期保持正向响应。说明短期内国家原油价格的正向冲击对原 油进口国存在负向影响, 对原油出口国存在正向影响, 长期内国际原油价格的正向冲击对各 国经济增长存在正向影响, 对于原油出口国, 该影响会持续较长时间。主要原因在于上海合 作组织相关国家中中国、印度等国作为主要的原油进口国, 国际油价上涨很大程度上增加了 国家财政支出, 短期内阻碍国民经济正常运行, 长期内国际油价倒逼国家产业结构和生产方 式进行变革, 以适应高油价的大环境, 所以长期内原油进口国国家经济发展逐渐恢复, 而俄 罗斯、哈萨克斯坦、乌兹别克斯坦以及伊朗拥有丰富的石油和天然气资源, 是重要的油气出 口国, 特别是俄罗斯与伊朗是世界排名靠前的原油出口大国, 国际油价上涨提高了国家出口 额, 对各国经济增长具有明显的促进作用。

第二, 汇率对国际原油价格冲击的反应。图 2-1 中的（e1）显示, 原油进口国汇率在受到国 际油价的正向冲击后, 当期产生不显著的正向响应, 滞后第 2 期开始, 正向响应逐渐增强, 到滞后第 4 期时响应保持平稳; 从图 2-2 中的 (d2) 可见, 受到国际油价的正向冲击, 原油 出口国汇率变化与原油进口国相似, 但是响应程度更加显著。说明国际油价的正向冲击对汇 率的影响较为波动, 不同时期的响应程度不同, 当国际油价上涨时, 本国货币汇率不断上升, 说明美元贬值, 本币升值, 原因在于通常原油出口国会接受美元结算, 随后再卖掉这些美元, 增加了外汇市场上美元供给, 这意味着国际油价上涨会导致美元贬值, 本币升值, 反之国际 油价下跌会导致美元升值, 本币贬值。对于以石油和天然气出口收入为主要财政收入来源的 俄罗斯来说, 国际油价暴跌不仅使其经济遭遇重创, 也使卢布遭遇重挫。如 2014 年 11 月 27 日 $0 P E C$ 宣布维持 2015 年石油生产目标 3000 万桶/日不变后, 两日内国际原油价格累计跌幅 近 10\%, 与之相对应的是俄罗斯货币卢布汇率 12 月 1 日暴跌, 下跌幅度一度达 $9 \%$, 创下自 1998 年金融危机以来的单日最大跌幅。

第三, 国际原油价格对汇率冲击的反应。从图 2-1 的 (c1) 和图 2-2 的 (b2) 可以看出, 汇 率的正向冲击对国际油价造成正面影响, 随着滞后期的增加, 这种正面影响快速增强后慢慢 减弱并趋于稳定, 说明汇率对国际油价具有微弱的正面影响, 并且具有一定时滞性。美元作 为目前国际原油价格交易的主要结算货币, 对国际原油价格的影响日渐增强, 并且美元汇率 的变动会直接影响上海合作组织相关国家的本币汇率。当汇率上升, 即美元贬值, 本币升值, 原油出口国会由于美元贬值而导致收入受损, 有推高油价的动机, 因此, 国际原油价格与汇 率之间存在相互的正向影响关系。

\section{4. 结论}

本文通过本币-美元汇率将国际原油价格内生化, 系统研究国际油价变动对上海合作组织相关 国家经济发展的影响。上述实证的结果表明国际原油价格的变动会给上海合作组织成员国与 观察员国的经济增长带来显著的影响, 从总量上来看, 更多的表现为直接的短期的效应。由 脉冲响应和方差分解结果可以看出, 短期内国际油价下降对上合组织内原油进口国的影响为 正向, 对原油出口国经济发展则产生负面影响, 并且国际油价波动是影响原油出口国国家宏 观经济的重要因素。对中国、印度等原油进口国而言, 国际原油价格的下降可以带来利好, 可以减少这些国家生产和消费成本, 原油作为重要的生产原料, 其价格下跌使下游产业及其 他主要工业制成品行业的生产成本大幅度下降, 推动成品油、钢材等大宗商品价格下跌, 大 大降低了生产和消费的成本, 提高生产的利润率, 有利于经济的稳定和增长。对于上海合作 组织相关国家中的俄罗斯、中亚四国、伊朗等原油生产和出口国来说, 国际油价下跌无疑会 在短期之内造成经济的困难。在产量既定的条件下，国际原油价格下跌会造成原油出口收入 的减少, 贸易逆差的风险增大, 尤其是以原油天然气产业为国民经济支柱的国家或地区将会 遭受沉重的打击。 
由脉冲响应和方差分解结果可以还可以看出, 国际原油价格与汇率存在相互促进的关系。目 前在国际原油贸易中美元作为主要的计价与结算货币, 各个国家本币与美元的汇率机制对原 油的交易价格形成了影响, 原油价格反过来也会影响本国汇率的变动。当原油价格下跌时, 美元产生升值的趋势, 其他国家货币则相对贬值, 在一定程度上会给其他国家经济发展带来 不利影响，加上美国调整其货币政策和地缘政治因素，退出量化宽松货币政策进一步促进美 元升值, 通过汇率传导机制引起其他国家宏观经济的变动。对于俄罗斯、中亚四国、伊朗等 原油出口国而言, 本国货币汇率下跌将给国民经济带来一连串负面打击, 实际汇率下跌将导 致本国货币购买力下降，居民面临物价飞涨，通货膨胀加剧的塩筴局面，并且国外投资者不 愿承担汇率下降带来的投资风险，国内投资总额急剧减少，导致国民经济下滑。对于中国、 印度等原油进口国而言, 美元升值, 本币相对贬值不利于原油进口, 需要支付更多美元来保 障国内原油供给, 从而减少对原油的需求, 会抵消一部分由原油价格下降所带来的原油需求 增加量。

从长期来看, 国际原油价格的波动会迫使各国调整经济结构, 以适应能源市场价格机制对本 国经济的常态化影响, 达到优化产业结构的目的。俄罗斯、中亚四国等以能源生产和出口作 为国家财政收入主要来源的国家为防止原油价格波动对本国经济的冲击, 会调整自身的经济 结构, 资本、劳动力等生产要素从能源密集型部门转移到其他产业部门, 厂商需要调整生产 经营策略以应对油价下跌带来的诸多负面冲击, 这将是一个长期的过程, 并且产生大量的调 整成本，会影响国民经济增长的速度和规模。对于上海合作组织中的原油进口国而言，原油 价格下跌将导致能源密集部门扩张, 这带来资本、劳动力等资源在产业部门之间的重新配置, 而这种调整也同样会带来社会调整成本的增加，并且不是短期之内可以实现的，同时大量的 能源消耗也会给长期的产能水平和环境保护带来巨大压力。与此同时，上海合作组织逐步推 动人民币和卢布国际化也是必要的, 改变现有的原油-美元结算体系和国际大宗商品(如原油) 的定价机制，以消除未来国际油价持续波动对上海合作组织带来的恶劣影响。

\section{参考文献}

[1] Hamilton, James D. Nonlinearities and The Macroeconomic Effects Of Oil Prices[J]. Macroeconomic Dynamics, (2011), No.15, pp. 364-378.

[2] Holtz-Eakin D. W. Newey, H. Rosen. Estimating Vector Auto regressions with Panel Data[J]. Econometrical, Vol. 56(1998), No.6, pp. 1371-1395.

[3] 郭晓琼. 世界经济危机深化背景下的上海合作组织 [J]. 俄罗斯中亚东欧研究, 2012, (5):63-71.

[4] 殷红．俄罗斯经济形势与政策分析[J]．俄罗斯东欧中亚研究，2014，(3):31-36.

[5] 张猛, 丁振辉. 上海合作组织自由贸易区:构想及其意义 [J]. 国际经贸探索, 2013, (2):22-33.

[6] 胡鞍钢, 马伟, 鄢一龙. “丝绸之路经济带”: 战略内涵、定位和实现路径 [J]. 新疆师范 大学学报(哲学社会科学版), 2014, (2):1-11.

[7] 李琪. 中国与中亚创新合作模式、共建“丝绸之路经济带”的地缘战略意涵和实践 [J]. 陕 西师范大学学报(哲学社会科学版), 2014, (4):5-15.

[8] 朱显平，张建政. “上海合作组织”框架下的中国与中亚国家金融合作目标及途径 $[\mathrm{J}]$. 东 北亚论坛，2007，(3):15-19.

[9] 耿晔强，马志敏. 基于博弯视角下的中国与上海合作组织成员国能源合作分析 $[\mathrm{J}]$. 世界 经济研究，2011，(5):82-86.

[10]刘乾, 周础. 上合组织框架下多边能源合作机制与中国的参与策略 $[\mathrm{J}]$. 中国原油大学学 报（社会科学版），2013，29(6):1-7. 


\section{References}

[1] Hamilton, James D. Nonlinearities and The Macroeconomic Effects Of Oil Prices[J]. Macroeconomic Dynamics, (2011), No.15, pp. 364-378.

[2] Holtz-Eakin D.,W. Newey, H. Rosen. Estimating Vector Auto regressions with Panel Data[J]. Econometrical, Vol. 56(1998), No.6, pp. 1371-1395.

[3] Guo Xiao-qiong: "Deepening world economic crisis in the context of the Shanghai Cooperation Organization"[J]. Russia Central East European Studies, (2012),No.5,p.63-71(in Chinese)

[4] Yin Hong: "Russian Economic Situation and Policy Analysis"[J]. Russia Central East European Studies, (2014),No.3,p.31-36(in Chinese)

[5] Zhang Meng, Ding Zhen-hui: "Shanghai Cooperation Organization free trade zone: Concept and Its Significance"'[J]. International Economics and Trade Research, (2013),No.2,p.22-33(in Chinese)

[6] Hu An-gang, Ma Wei, Yan Yi-long: “'Silk Road Economic Belt':strategic content, positioning and implementation path"[J]. Xinjiang Normal University (Philosophy and Social Sciences), (2014),No.2,p.1-11(in Chinese)

[7] Li Qi: "Geo-strategic and practical implications of new modes of cooperation between China and Central Asia, to build a 'Silk Road Economic Belt"'[J]. Shaanxi Normal University (Philosophy and Social Sciences), (2014),No.4,p.5-15(in Chinese)

[8] Zhu Xian-ping, Zhang Jian-zheng: "China and Central Asian countries under financial cooperation objectives and the ways of the 'Shanghai Cooperation Organization' Framework"[J]. Northeast Asia Forum, (2007),No.3,p.15-19(in Chinese)

[9] Geng Ye-ping, Ma Zhi-min: "Member States Energy Cooperation of China and the Shanghai Cooperation Organization Based on Game Perspective"[J]. World Economy, (2011),No.5,p.82-86(in Chinese)

[10]Liu Qi, Zhou Chu: "SCO Multilateral Energy Cooperation Mechanism and China's participation in the policy framework" [J]. China's crude oil University (Social Sciences), Vol.29 (2013),No.6,p. 1-7(in Chinese)

作者简介: 王宇(1992年-), 女, 河南省新乡市, 主要研究方向: 产业经济, 邮箱: wymjhy@126. com 